\title{
Development and Evaluation of Alprazolam Controlled Release tablets
}

\author{
Sarmila Shrestha (Amatya) ${ }^{1}$, \\ Dharma Prasad Khanal ${ }^{2}$ \\ \& Panna Thapa ${ }^{3}$
}

\begin{abstract}
Twenty three different tablet formulations of alprazolam were prepared using Polymer like hydroxypropylmethyl cellulose (HPMC K4M, HPMC K15M and HPMC K100M) in the concentration of $5-50 \%$ of total weight of tablets and combination of HPMC K15M and HPMC K100M with ethyl cellulose (EC) was formulated by using wet granulation method. Drug formulation containing $1.0 \mathrm{mg}, 1.5 \mathrm{mg}, 5 \mathrm{mg}, 10 \mathrm{mg}$ and $15 \mathrm{mg}$ alprazolam per tablet maintaining constant HPMC K15M concentration was also developed.

The in-vitro dissolution studies of the formulated and marketed product in USP type II apparatus showed that the drug release is dependent upon the drug: polymer ratio; also molecular weight of the polymer and solubility of loaded drug. With increasing concentration and molecular weight of polymer, drug release was found to be decreased. When formulating the tablets the method used whether direct compression or wet granulations also affect the release of the drug from matrix. Wet granulation method by using $40 \%$ HPMC K15M in combination with $5 \% \mathrm{EC}$ was found to be most suitable controlled release alprazolam tablet as drug release was found to be appreciable in this formulation. When loading dose of alprazolam was increased, drug release was found to be tremendously decreased because of the poor solubility of alprazolam in water. When one-way ANOVA was applied for various formulated and marketed tablets it was found that there is no significant difference $(p>0.05)$ in drug release rate among formulation similarly model independent methods was also applied such as similarity and dissimilarity factor and found that there is no significant difference between these formulations. Key Words: Controlled release, Alprazolam, Polymers, Dissolution, HPMC.
\end{abstract}

\section{Introduction}

Oral drug delivery has been known for decades as the most widely utilized route of administration among all the routes that have been explored for the systemic delivery of drugs via various pharmaceuticals products of different dosage forms. The reasons that the oral route achieved such popularity may be in part attributed to its ease of administration, patient compliance, and cost effectiveness [1].

Controlled release dosage forms (CRDF) can be defined as prolonged action formulations, which provide continuous release of medicaments at a predetermined rate and predetermined time [2]. The main advantages of controlled drug delivery systems are maintenance of therapeutically optimum drug concentrations in the plasma through zero order release without

\footnotetext{
${ }^{1}$ Corresponding Author: amatyaz@ hotmail.com

${ }^{2}$ M.Pharm Ph.D, GM,Lomus Pharmaceuticals Pvt.Ltd

${ }^{3}$ M. Pharm Ph.D, HoD, Department of Pharmacy, Kathmandu university.
}

Department of pharmacy, Kathmandu University, Dhulikhel, Nepal, GPO Box 6250 (Kathmandu) 
significant fluctuations and elimination of the need for frequent single dose administrations [3]. Different types of CRDF systems are prepared depending on this behavior of the polymers and the method of drug release from these polymers differs upon its design and its physiochemical characteristics. Alprazolam is a short-acting drug in the benzodiazepine class used to treat anxiety disorders and as an adjunctive treatment for depression [4].

Pharmacokinetic data: Bioavailability- 80-90\%, Metabolism - Hepatic, Half-life - 6-12 hours and Excretion- Renal

Alprazolam is readily absorbed from the gastrointestinal tract. The peak plasma concentration is achieved in 1-2 hours. Most of the drug is bound to plasma protein, mainly albumin.

Controlled drug delivery occurs when a polymer, whether natural or synthetic, is judiciously combined with a drug or other active agent in such a way that the active agent is released from the material in a pre-designed manner. Because of low costs and ease of fabrication, one of the most common approaches to get controlled release is to embed a drug in a hydrophobic matrix such as wax, polyethylene, polypropylene, and ethyl cellulose or hydrophilic matrix such as hydroxypropylcellulose, HPMC, methylcellulose, sodium carboxymethyl cellulose (sodium CMC) [5]

Polymer used in the formulation of controlled release Alprazolam is HPMC of various grades (HPMC-K4M, HPMC- K15M and HPMC- K100M) and EC. The use of hydrophilic polymers in preparation of $\mathrm{CRDF}$ is considered as simple because of its gel forming properties in contact with water. The gel formation of the polymer is the barrier for controlling drug transport from the dosage form to outer environment. In addition the integrity of polymer in water and its gel strength is dependent upon its molecular size, polymer relaxation temperature and hydrophilicity of the polymer [6].

As water insoluble excipients, EC can effectively control the release of an active by modifying the size and length of the diffusion path. In this role, it is typically used in combination with a water-soluble active, water-soluble excipient such as a HPMC cellulose ether and poly ethylen glycol (PEG) . By varying excipient ratio and the particle size, a wide variety of release rate profiles can be achieved [7] [8] [9].

\section{Materials and Methods}

\section{Materials}

Alprazolam (Chacko suns chemicals, India), HPMC K4M, HPMC K15 M (Feicheng Ruitai fine chemicals, China), Ethyl Cellulose (Samsung fine chemicals, Shaghai China), Starch (Shital chemicals, India), Microcrystalline Cellulose Powder PH 101 [(MCCP)(Sigachi Chemicals India)], Magnesium Stearate (Varsh Chemicals, India), Purified Talc (Niclon Chemicals, India), Aerosil (Rutocel, Beijing), were obtained from Lomus Pharmaceuticals Pvt. Ltd. (Gothatar, Kathmandu Nepal) as a gift sample. HPMC K100M (Dow chemicals) was obtained from Research Lab of Pharmacy department, Kathmandu University, Dhulikhel Nepal and Market Product (Alpras, Torrent India) was purchased from Delhi, India and coded as RT.

\section{Equipments and Instrument}

Tray Drier - Global Enterprises, India, Tablet Compression machine: 16 stations rotary tablet compression machine - Cadmach India, Balance for dispensing - OHAUS , USA, Analytical Balance - Scaltec Germany, UV Spectrophotometer - Shimadzu, Japan ( UV - 2450), USP Dissolution Apparatus - Electro lab, Moisture Balance - Scaltech Germany, Friability Tester - Electro lab Friabilator, Hardness Tester - Campbell, India, Vernier Caliper - Mitutoya Corporation, Japan. 


\section{Method}

\section{Formulation by Dry granulation method}

Two different formulations using Dry granulation method were prepared. For each batch Polymer was passed through 40\#. Starch and MCCP were passed through 60 \#. Alprazolam, excipients and polymer was mixed manually in SS tray for 15 minutes which was passed through 14 \#. The powder so obtained was mixed with lubricants (Magnesium Stearate, Purified Talc) which were initially passed through 100 \#. Then the powder blend was mixed in poly bag for 10 minutes and finally compressed in tablet compression machine with die size of $8.0 \mathrm{~mm}$. Content uniformity test was performed and found to be out of limit so invitro dissolution test was not done. The results are summarized in the Table2.

\section{Formulation by nonaqueous granulation method}

Twenty-one different formulations using wet granulation method were prepared. For each batch Polymer was passed through 40\#. Starch and MCCP were passed through 60 \#. Alprazolam was dissolved in alcohol and the polymer were dispersed in methylene chloride Then the drug, excipients and polymer was mixed manually in stainless steel (SS) tray for 15 minutes to form a wet dough mass which was passed through 14 \#. The granules so obtained were initially dried in air for 5 minutes and semi-dried granules were passed through 20 \# followed by final drying at $50{ }^{\circ} \mathrm{C}$ in tray drier for 15 minutes. The Purified Talc, Magnesium Stearate, was sieved through 100 \#. Then the lubricants was added to the granules and mixed in double lined poly-bag for 10 minutes. Finally granules were compressed in tablet compression machine with die size of $8.0 \mathrm{~mm}$. The tablets were tested for the physical characteristics and in vitro dissolution rate. Composition for formulation of Alprazolam tablet by using hydrophilic polymer and combination of hydrophilic and hydrophobic polymer are summarized in Table1.

Table 1(a)

\begin{tabular}{|c|c|c|c|c|c|c|c|c|c|c|c|c|}
\hline \multicolumn{13}{|c|}{$\begin{array}{l}\text { Composition of Controlled release Alprazolam } \\
\text { Tablets }\end{array}$} \\
\hline $\begin{array}{l}\text { Ingredients } \\
(\mathrm{mg} / \mathrm{tab})\end{array}$ & $\begin{array}{l}\text { ACR- } \\
01\end{array}$ & $\begin{array}{l}\text { ACR- } \\
02\end{array}$ & ACR-03 & ACR-04 & $\begin{array}{l}\text { ACR- } \\
05\end{array}$ & $\begin{array}{l}\text { ACR- } \\
06\end{array}$ & ACR-07 & ACR-08 & ACR-09 & ACR-10 & ACR-11 & $\begin{array}{l}\text { ACR- } \\
12\end{array}$ \\
\hline Alprazolam & 1.5 & 1.5 & 1.5 & 1.5 & 1.5 & 1.5 & 1.5 & 1.5 & 1.5 & 1.5 & 1.5 & 1.5 \\
\hline Alcohol $(\mu \mathrm{l})$ & 10 & 10 & 10 & 10 & 10 & 10 & 10 & 10 & 10 & 10 & 10 & 10 \\
\hline Starch & 157 & 157 & 157 & 145.5 & 145.5 & 145.5 & 122.5 & 122.5 & 122.5 & 99.5 & 99.5 & 76.5 \\
\hline МССР РН 101 & 50 & 50 & 50 & 50 & 50 & 50 & 50 & 50 & 50 & 50 & 50 & 50 \\
\hline HPMC K4M & 11.5 & - & - & 23 & - & - & 46 & - & - & - & - & - \\
\hline HPMC K15M & - & 11.5 & - & - & 23 & - & - & 46 & - & 69 & - & 92 \\
\hline HPMC K100M & - & - & 11.5 & - & - & 23 & - & - & 46 & - & 69 & - \\
\hline Ethyl Cellulose & - & - & - & - & - & - & - & - & - & 0 & 0 & 0 \\
\hline Purified Talc & 5 & 5 & 5 & 5 & 5 & 5 & 5 & 5 & 5 & 5 & 5 & 5 \\
\hline \multicolumn{13}{|l|}{ Magnesium } \\
\hline Stearate & 4 & 4 & 4 & 4 & 4 & 4 & 4 & 4 & 4 & 4 & 4 & 4 \\
\hline Aerosil-200 & 1 & 1 & 1 & 1 & 1 & 1 & 1 & 1 & 1 & 1 & 1 & 1 \\
\hline
\end{tabular}




\begin{tabular}{lc|llllllllllll}
\hline $\begin{array}{l}\text { Total } \\
(\mathrm{mg})\end{array}$ & weight & 230 & 230 & 230 & 230 & 230 & 230 & 230 & 230 & 230 & 230 & 230 & 230 \\
\hline
\end{tabular}

\begin{tabular}{|c|c|c|c|c|c|c|c|c|c|c|c|}
\hline $\begin{array}{l}\text { Composition of } \\
\text { tabets }\end{array}$ & ontrolled & release & Alprazolam & & & & & & & & \\
\hline $\begin{array}{l}\text { Ingredients } \\
(\mathrm{mg} / \mathrm{tab})\end{array}$ & $\begin{array}{l}\text { ACR- } \\
13\end{array}$ & $\begin{array}{l}\text { ACR- } \\
14\end{array}$ & ACR-15 & ACR-16 & $\begin{array}{l}\text { ACR- } \\
17\end{array}$ & $\begin{array}{l}\text { ACR- } \\
18\end{array}$ & ACR-19 & ACR-20 & ACR-21 & ACR-22 & ACR-23 \\
\hline Alprazolam & 1.5 & 1.5 & 1.5 & 1.5 & 1.5 & 1 & 5 & 10 & 15 & 1.5 & 1.5 \\
\hline Alcohol $(\mu \mathrm{l})$ & 10 & 10 & 10 & 10 & 10 & 7.5 & 37.5 & 75 & 112.5 & 10 & 10 \\
\hline Starch & 76.5 & 53.5 & 53.5 & 65 & 65 & 77 & 73 & 68 & 63 & 76.5 & 76.5 \\
\hline МССР РН 101 & 50 & 50 & 50 & 50 & 50 & 50 & 50 & 50 & 50 & 50 & 50 \\
\hline HPMC K4M & - & - & - & - & - & - & - & - & - & - & - \\
\hline HPMC K15M & - & 115 & - & 92 & - & 92 & 92 & 92 & 92 & 92 & 92 \\
\hline HPMC K100M & 92 & - & 115 & - & 92 & - & - & - & - & - & - \\
\hline Ethyl Cellulose & 0 & 0 & 0 & 11.5 & 11.5 & - & - & - & - & - & - \\
\hline Purified Talc & 5 & 5 & 5 & 5 & 5 & 5 & 5 & 5 & 5 & 5 & 5 \\
\hline \multicolumn{12}{|l|}{ Magnesium } \\
\hline Stearate & 4 & 4 & 4 & 4 & 4 & 4 & 4 & 4 & 4 & 4 & 4 \\
\hline Aerosil-200 & 1 & 1 & 1 & 1 & 1 & 1 & 1 & 1 & 1 & 1 & 1 \\
\hline $\begin{array}{l}\text { Total } \quad \text { weight } \\
(\mathrm{mg})\end{array}$ & 230 & 230 & 230 & 230 & 230 & 230 & 230 & 230 & 230 & 230 & 230 \\
\hline
\end{tabular}

\section{Evaluation of Tablets}

The tablets were evaluated for their physicochemical parameters such as weight variation, hardness, thickness, friability, assay, content uniformity, in-vitro dissolution.

\section{Assay Method}

Twenty tablets from each batch were sampled, weighed and crushed to a fine powder and quantity equivalent to $1.5 \mathrm{mg}$ of Alprazolam was dissolved in $25 \mathrm{ml}$ of $0.1 \mathrm{~N} \mathrm{HCl}$ solutions and volume was made $100 \mathrm{ml}$ with same solvent and sonicated for 15 minutes. Then the solutions were filtered through whatman filter paper and $10 \mathrm{ml}$ of solution was diluted to 25 $\mathrm{ml}$ with $0.1 \mathrm{~N} \mathrm{HCl}$ solutions. The absorbance of this final solution and the same concentration of standard Alprazolam solution were compared at wavelength $260 \mathrm{~nm}$. The results are summarized in the Table 2.

\section{Content Uniformity Test}

10 tablets from each batch were dissolved individually in $25 \mathrm{ml}$ of $0.1 \mathrm{~N} \mathrm{HCl}$ solutions and volume was made $100 \mathrm{ml}$ with same solvent and sonicated for 15 minutes. Solutions were filtered through whatman filter and $10 \mathrm{ml}$ of solution was diluted to $25 \mathrm{ml}$ with $0.1 \mathrm{~N} \mathrm{HCl}$. Standard solution of Alprazolam of same concentration was compared with sample solution at wavelength $260 \mathrm{~nm}$. The results are summarized in the Table 2.

\section{Dissolution Tests}


USP apparatus II was used to test the dissolution profiles of the reference product and formulated products in $500 \mathrm{ml} 0.1 \mathrm{~N} \mathrm{HCl}$ as medium at $37 \pm 0.5^{\circ} \mathrm{C}$ with paddle speed $50 \mathrm{rpm}$ [38]. $10 \mathrm{ml}$ of sample was drawn at every hour and equal volume of medium was replaced into each of the dissolution jars after each sampling to maintain the sink condition. Absorbance of each filtrate sample was measured and compared with the same concentration of standard solution in UV spectrophotometer at wavelength $260 \mathrm{~nm}$. The dissolution data of all the formulated and reference products are summarized in Table 3. 
Journal of Manmohan Memorial Institute of Health Sciences (JMMIHS)

Table 2 Physical characteristics (Mean \pm SD) of formulated controlled release Alprazolam tablets

\begin{tabular}{|c|c|c|c|c|c|c|c|c|c|c|c|c|c|c|c|c|c|c|c|}
\hline \multirow{2}{*}{$\begin{array}{l}\text { Formulation } \\
\text { ACR- } 01\end{array}$} & \multicolumn{3}{|c|}{$\begin{array}{l}\text { Average weight }{ }^{\mathrm{a}} \\
(\mathrm{mg})\end{array}$} & \multicolumn{3}{|c|}{$\begin{array}{l}\text { Hardness } \\
\left(\mathrm{Kg} / \mathrm{cm}^{2}\right)\end{array}$} & \multicolumn{3}{|c|}{$\begin{array}{l}\text { Thickness }^{\mathrm{a}} \\
(\mathrm{mm})\end{array}$} & \multicolumn{3}{|c|}{$\begin{array}{l}\text { Diameter }^{\mathrm{a}} \\
(\mathrm{mm})\end{array}$} & \multirow{2}{*}{$\begin{array}{l}\begin{array}{l}\text { Friability }^{\mathrm{a}} \\
(\%)\end{array} \\
0.06\end{array}$} & \multicolumn{3}{|l|}{$\begin{array}{l}\text { Assay }^{\mathrm{c}} \\
(\%)\end{array}$} & \multicolumn{3}{|c|}{$\begin{array}{l}\text { Content Uniformity } \\
(\%)\end{array}$} \\
\hline & 230.40 & \pm & 4.51 & 6.95 & \pm & 0.90 & 0.00 & \pm & 0.00 & 0.00 & \pm & 0.00 & & 96.32 & \pm & 1.50 & 100.40 & \pm & 3.59 \\
\hline ACR- 02 & 230.20 & \pm & 4.45 & 6.1 & \pm & 0.57 & 0.00 & \pm & 0.00 & 0.00 & \pm & 0.00 & 0.04 & 102.67 & \pm & 1.09 & 99.08 & \pm & 4.11 \\
\hline ACR- 03 & 230.05 & \pm & 3.22 & 5.65 & \pm & 0.53 & 0.00 & \pm & 0.00 & 0.00 & \pm & 0.00 & 0.16 & 101.64 & \pm & 0.96 & 100.18 & \pm & 4.20 \\
\hline ACR- 04 & 228.65 & \pm & 3.99 & 7.01 & \pm & 1.18 & 0.00 & \pm & 0.00 & 0.00 & \pm & 0.00 & 0.18 & 96.87 & \pm & 0.43 & 99.98 & \pm & 4.52 \\
\hline ACR- 05 & 230.55 & \pm & 3.49 & 5.75 & \pm & 0.54 & 0.00 & \pm & 0.00 & 0.00 & \pm & 0.00 & 0.09 & 97.45 & \pm & 0.47 & 98.84 & \pm & 2.78 \\
\hline ACR- 06 & 230.00 & \pm & 4.38 & 6.8 & \pm & 0.67 & 0.00 & \pm & 0.00 & 0.00 & \pm & 0.00 & 0.09 & 97.98 & \pm & 1.59 & 99.34 & \pm & 4.59 \\
\hline ACR- 07 & 229.45 & \pm & 3.64 & 6.46 & \pm & 0.59 & 0.00 & \pm & 0.00 & 0.00 & \pm & 0.00 & 0.15 & 100.88 & \pm & 0.77 & 99.34 & \pm & 4.34 \\
\hline ACR- 08 & 231.10 & \pm & 4.35 & 6.25 & \pm & 0.79 & 0.00 & \pm & 0.00 & 0.00 & \pm & 0.00 & 0.21 & 101.35 & \pm & 0.04 & 99.08 & \pm & 4.63 \\
\hline ACR- 09 & 229.80 & \pm & 3.33 & 7.15 & \pm & 0.75 & 0.00 & \pm & 0.00 & 0.00 & \pm & 0.00 & 0.04 & 97.91 & \pm & 0.64 & 101.44 & \pm & 4.52 \\
\hline ACR- 10 & 229.40 & \pm & 3.34 & 7.1 & \pm & 0.88 & 0.00 & \pm & 0.00 & 0.00 & \pm & 0.00 & 0.19 & 97.24 & \pm & 0.48 & 99.69 & \pm & 3.47 \\
\hline ACR- 11 & 230.75 & \pm & 2.57 & 7 & \pm & 0.85 & 0.00 & \pm & 0.00 & 0.00 & \pm & 0.00 & 0.07 & 97.74 & \pm & 1.16 & 101.13 & \pm & 5.08 \\
\hline ACR- 12 & 230.65 & \pm & 4.10 & 7.75 & \pm & 0.63 & 0.00 & \pm & 0.00 & 0.00 & \pm & 0.00 & 0.11 & 98.97 & \pm & 1.29 & 98.49 & \pm & 4.90 \\
\hline ACR- 13 & 230.50 & \pm & 2.27 & 7.8 & \pm & 0.54 & 0.00 & \pm & 0.00 & 0.00 & \pm & 0.00 & 0.27 & 99.48 & \pm & 0.53 & 97.97 & \pm & 4.20 \\
\hline ACR- 14 & 229.05 & \pm & 3.85 & 7.95 & \pm & 0.50 & 0.00 & \pm & 0.00 & 0.00 & \pm & 0.00 & 0.12 & 99.40 & \pm & 2.41 & 100.41 & \pm & 4.61 \\
\hline ACR- 15 & 229.50 & \pm & 4.20 & 7.15 & \pm & 0.67 & 0.00 & \pm & 0.00 & 0.00 & \pm & 0.00 & 0.064 & 97.26 & \pm & 0.63 & 100.05 & \pm & 3.08 \\
\hline ACR- 16 & 228.95 & \pm & 3.44 & 8.5 & \pm & 0.53 & 0.00 & \pm & 0.00 & 0.00 & \pm & 0.00 & 0.04 & 98.08 & \pm & 1.47 & 98.03 & \pm & 4.55 \\
\hline ACR- 17 & 229.45 & \pm & 4.06 & 8.55 & \pm & 0.55 & 0.00 & \pm & 0.00 & 0.00 & \pm & 0.00 & 0.03 & 99.99 & \pm & 1.51 & 100.39 & \pm & 4.39 \\
\hline ACR- 18 & 229.00 & \pm & 1.63 & 8.4 & \pm & 0.52 & 0.00 & \pm & 0.00 & 0.00 & \pm & 0.00 & 0.13 & 99.26 & \pm & 2.56 & 100.33 & \pm & 2.06 \\
\hline ACR- 19 & 229.00 & \pm & 1.87 & 8.4 & \pm & 0.46 & 0.00 & \pm & 0.00 & 0.00 & \pm & 0.00 & 0.09 & 99.09 & \pm & 2.11 & 99.51 & \pm & 2.93 \\
\hline ACR- 20 & 230.00 & \pm & 1.47 & 8.45 & \pm & 0.44 & 0.00 & \pm & 0.00 & 0.00 & \pm & 0.00 & 0.07 & 97.97 & \pm & 1.54 & 100.21 & \pm & 3.51 \\
\hline ACR- 21 & 226.00 & \pm & 1.65 & 8.2 & \pm & 0.63 & 0.00 & \pm & 0.00 & 0.00 & \pm & 0.00 & 0.06 & 97.39 & \pm & 0.86 & 100.14 & \pm & 1.87 \\
\hline ACR- 22 & 230.00 & \pm & 2.02 & 9.1 & \pm & 0.46 & 0.00 & \pm & 0.00 & 0.00 & \pm & 0.00 & 0.14 & 106.57 & \pm & 2.74 & 108.57 & \pm & 22.53 \\
\hline ACR- 23 & 231.00 & \pm & 2.04 & 9 & \pm & 0.47 & 0.00 & \pm & 0.00 & 0.00 & \pm & 0.00 & 0.18 & 98.01 & \pm & 1.52 & 107.00 & \pm & 26.12 \\
\hline
\end{tabular}




\section{Mathematical Modeling of Drug Release Profile}

The dissolution data obtained for formulated products and reference product were analyzed using various mathematical models.

\section{Zero Order Kinetics}

Zero order reaction is defined as a reaction in which the rate does not depend on the concentration terms of reactant. This is the ideal method of drug release to achieve prolonged pharmacological action. The equation for zero order kinetic is represented as follows,

$$
\mathrm{Q}_{1}=\mathrm{Q}_{0}+\mathrm{k}_{\mathrm{o}} \mathrm{t}
$$

Where, $\mathrm{Q}_{1=}$ Amount of drug dissolved in time $\mathrm{t}$, and

$\mathrm{Q}_{0}=$ Initial amount of drug in the solution, which is often zero and $\mathrm{K}_{\mathrm{o}}$ is the zero order release constant. Average of Qo- $Q_{1}$ was calculated and a graph of Qo- $Q_{1}$ versus time 't' was plotted for each formulation.

\section{First Order Kinetic Model}

First order process is defined as a reaction in which the rate of reaction depends on the concentration of one reactant. i.e. greater the concentration, faster the reaction. The pharmaceutical dosage forms containing water-soluble drugs in porous matrices follow first order release kinetics, and can be expressed by the equation

$$
\log Q_{1}=\log Q_{0}+\frac{k t}{2.303}
$$

Here also, average of $\log \mathrm{Q}_{\mathrm{o}} / \mathrm{Q}_{\mathrm{t}}$ was calculated and graph of $\log \mathrm{Q}_{\mathrm{o}} / \mathrm{Q}_{\mathrm{t}}$ versus time ' $\mathrm{t}$ ' was plotted for formulation, which show 12 hours release. The degree of correlation $\left(\mathrm{R}^{2}\right)$ obtained after passing the trend line with y-intercept which was then used to determine whether or not the release from the different formulation are following the first order or not.

\section{Higuchi and Korsmeyer - Peppas Model}

Both the Higuchi model and Korsmeyer - Peppas model is obtained from power law proposed by Ritger and Peppas as stated in equation (3);

$$
\frac{\mathbf{M}_{\mathrm{t}}}{\mathbf{M}}=\mathbf{k t}^{\mathrm{n}}
$$

The fitness of data to Higuchi equation was assessed by determining the correlation coefficient between square root of time and $M_{t} / M_{\propto}$. Plot of $M_{t} / M_{\propto}$ versus $t^{1 / 2}$ gives a straight line with a slop of $\mathrm{k}_{\mathrm{H}}$, Higuchi dissolution rate constant.The fitness of data to Korsmeyer - Peppas model was assessed by determining the correlation coefficient between $\log \left(\mathrm{M}_{\mathrm{t}} / \mathrm{M}_{\propto}\right)$ and $\log$ of time, $\mathrm{t}$. Plot of $\log \left(\mathrm{M}_{\mathrm{t}} / \mathrm{M}_{\propto}\right)$ versus $\log$ of time till the $60 \%$ of drug is released will be a straight line with a slope of $n$ and the intercept value of $\log \mathrm{k}$. anti-log of $\log \mathrm{k}$ gives the value of k. " $\mathrm{n}$ " is release exponent of different geometrical symmetry. 
Table 3(a) In-Vitro Dissolution of formualted and marketed controlled release Alprazolam tablets

\begin{tabular}{|c|c|c|c|c|c|c|c|c|c|c|c|c|c|c|c|c|c|c|c|c|c|c|}
\hline \multirow{2}{*}{$\begin{array}{l}\text { Alprazolam } \\
(\mathrm{mg})\end{array}$} & \multicolumn{4}{|c|}{ Polymer Content } & \multicolumn{18}{|c|}{$\begin{array}{l}\text { Cumulative \% of Drug Released (Mean } \pm \mathrm{SD} ; \mathrm{n}=6 \text { ) } \\
\text { (hour) }\end{array}$} \\
\hline & Grade & $(\%)$ & $\frac{\mathrm{EC}}{(\%)}$ & Formulation & 1 & & & 2 & & & 3 & & & 4 & & & 5 & & & 6 & & \\
\hline 1.5 & K4M & 5 & - & ACR- 01 & 89.23 & \pm & 0.39 & 96.13 & \pm & 1.05 & & & & & & & & & & & & \\
\hline 1.5 & $\mathrm{~K} 15 \mathrm{M}$ & 5 & - & ACR- 02 & 87.68 & \pm & 0.88 & 95.48 & \pm & 0.65 & & & & & & & & & & & & \\
\hline 1.5 & K100M & 5 & - & ACR- 03 & 85.32 & \pm & 0.67 & 95.10 & \pm & 1.33 & & & & & & & & & & & & \\
\hline 1.5 & $\mathrm{~K} 4 \mathrm{M}$ & 10 & - & ACR- 04 & 70.83 & \pm & 1.19 & 78.24 & \pm & 0.71 & 87.52 & \pm & 0.98 & 97.80 & \pm & 1.57 & & & & & & \\
\hline 1.5 & $\mathrm{~K} 15 \mathrm{M}$ & 10 & - & ACR- 05 & 68.15 & \pm & 0.80 & 74.23 & \pm & 1.41 & 86.01 & \pm & 0.18 & 95.11 & \pm & 1.17 & & & & & & \\
\hline 1.5 & K100M & 10 & - & ACR- 06 & 67.01 & \pm & 1.16 & 76.92 & \pm & 1.61 & 84.92 & \pm & 1.36 & 94.82 & \pm & 1.15 & & & & & & \\
\hline 1.5 & $\mathrm{~K} 4 \mathrm{M}$ & 20 & - & ACR- 07 & 62.11 & \pm & 0.89 & 69.80 & \pm & 0.64 & 76.60 & \pm & 0.91 & 80.92 & \pm & 0.72 & 88.32 & \pm & 0.32 & 96.02 & \pm & 0.26 \\
\hline 1.5 & $\mathrm{~K} 15 \mathrm{M}$ & 20 & - & ACR- 08 & 56.03 & \pm & 1.25 & 64.19 & \pm & 0.95 & 76.30 & \pm & 0.85 & 78.19 & \pm & 0.52 & 83.17 & \pm & 0.49 & 95.07 & \pm & 0.85 \\
\hline 1.5 & K100M & 20 & - & ACR- 09 & 53.33 & \pm & 0.82 & 62.18 & \pm & 1.28 & 75.09 & \pm & 0.70 & 76.35 & \pm & 1.33 & 81.63 & \pm & 0.80 & 93.19 & \pm & 2.60 \\
\hline 1.5 & $\mathrm{~K} 15 \mathrm{M}$ & 30 & - & ACR- 10 & 47.60 & \pm & 1.17 & 56.12 & \pm & 0.67 & 63.31 & \pm & 1.48 & 69.99 & \pm & 0.77 & 76.43 & \pm & 1.03 & 80.29 & \pm & 1.20 \\
\hline 1.5 & K100M & 30 & - & ACR- 11 & 45.04 & \pm & 0.96 & 52.12 & \pm & 1.50 & 60.01 & \pm & 0.80 & 67.16 & \pm & 0.21 & 73.14 & \pm & 0.73 & 79.32 & \pm & 1.81 \\
\hline 1.5 & $\mathrm{~K} 15 \mathrm{M}$ & 40 & - & ACR- 12 & 31.11 & \pm & 1.02 & 47.38 & \pm & 0.76 & 56.16 & \pm & 1.29 & 67.20 & \pm & 0.94 & 73.39 & \pm & 0.36 & 77.27 & \pm & 0.21 \\
\hline 1.5 & $\mathrm{~K} 100 \mathrm{M}$ & 40 & - & ACR- 13 & 30.20 & \pm & 0.95 & 45.29 & \pm & 0.64 & 55.32 & \pm & 0.54 & 64.13 & \pm & 0.61 & 73.01 & \pm & 0.71 & 76.16 & \pm & 1.99 \\
\hline 1.5 & $\mathrm{~K} 15 \mathrm{M}$ & 50 & - & ACR- 14 & 23.11 & \pm & 1.01 & 30.15 & \pm & 0.42 & 39.93 & \pm & 1.37 & 40.17 & \pm & 1.39 & 48.17 & \pm & 0.45 & 50.93 & \pm & 0.74 \\
\hline 1.5 & K100M & 50 & - & ACR- 15 & 21.09 & \pm & 1.06 & 29.66 & \pm & 0.94 & 37.18 & \pm & 0.85 & 40.09 & \pm & 1.26 & 47.09 & \pm & 0.08 & 48.63 & \pm & 1.09 \\
\hline 1.5 & $\mathrm{~K} 15 \mathrm{M}$ & 40 & 5 & ACR- 16 & 29.70 & \pm & 0.71 & 45.36 & \pm & 0.50 & 51.23 & \pm & 0.49 & 59.08 & \pm & 1.33 & 63.09 & \pm & 2.67 & 68.43 & \pm & 1.03 \\
\hline 1.5 & $\mathrm{~K} 100 \mathrm{M}$ & 40 & 5 & ACR- 17 & 28.36 & \pm & 0.93 & 43.15 & \pm & 0.74 & 49.15 & \pm & 0.93 & 57.33 & \pm & 1.20 & 61.11 & \pm & 1.40 & 66.39 & \pm & 1.45 \\
\hline 1 & $\mathrm{~K} 15 \mathrm{M}$ & 40 & - & ACR- 18 & 29.13 & \pm & 1.43 & 45.38 & \pm & 0.98 & 53.77 & \pm & 0.86 & 63.74 & \pm & 1.08 & 70.43 & \pm & 4.05 & 74.21 & \pm & 0.24 \\
\hline 5 & $\mathrm{~K} 15 \mathrm{M}$ & 40 & - & ACR- 19 & 10.78 & \pm & 1.24 & 18.29 & \pm & 1.03 & 21.66 & \pm & 0.76 & 26.80 & \pm & 0.99 & 33.56 & \pm & 1.84 & 44.32 & \pm & 0.40 \\
\hline 10 & $\mathrm{~K} 15 \mathrm{M}$ & 40 & - & ACR- 20 & 0.00 & \pm & 0.00 & 0.00 & \pm & 0.00 & 13.41 & \pm & 1.05 & 15.12 & \pm & 0.79 & 18.68 & \pm & 0.76 & 23.96 & \pm & 0.77 \\
\hline 15 & $\mathrm{~K} 15 \mathrm{M}$ & 40 & - & ACR- 21 & 0.00 & \pm & 0.00 & 0.00 & \pm & 0.00 & 0.00 & \pm & 0.00 & 0.00 & \pm & 0.00 & 10.64 & \pm & 0.87 & 15.40 & \pm & 1.08 \\
\hline 1.5 & - & - & - & RT & 25.89 & \pm & 1.10 & 40.15 & \pm & 1.67 & 51.67 & \pm & 0.95 & 54.32 & \pm & 0.72 & 62.05 & \pm & 0.52 & 69.29 & \pm & 1.00 \\
\hline
\end{tabular}

Table 3 (b) In-Vitro Dissolution of formulated and marketed controlled release Alprazolam tablets 
Journal of Manmohan Memorial Institute of Health Sciences (JMMIHS)

\begin{tabular}{|c|c|c|c|c|c|c|c|c|c|c|c|c|c|c|c|c|c|c|c|c|c|c|}
\hline \multirow{3}{*}{$\begin{array}{l}\text { Alprazolam } \\
(\mathrm{mg})\end{array}$} & \multicolumn{4}{|c|}{ Polymer Content } & \multirow{2}{*}{\multicolumn{18}{|c|}{$\begin{array}{l}\text { Cumulative } \% \text { of Drug Released (Mean } \pm \text { SD; } n=6 \text { ) } \\
\text { (hour) }\end{array}$}} \\
\hline & \multicolumn{2}{|l|}{ HPMC } & \multirow{2}{*}{$\frac{\mathrm{EC}}{(\%)}$} & \multirow[t]{2}{*}{ Formulation } & & & & & & & & & & & & & & & & & & \\
\hline & Grade & $(\%)$ & & & 7 & & & 8 & & & 9 & & & 10 & & & 11 & & & 12 & & \\
\hline 1.5 & $\mathrm{~K} 4 \mathrm{M}$ & 5 & - & ACR- 01 & & & & & & & & & & & & & & & & & & \\
\hline 1.5 & $\mathrm{~K} 15 \mathrm{M}$ & 5 & - & ACR- 02 & & & & $\mathrm{~s}$ & & & & & & & & & & & & & & \\
\hline 1.5 & K100M & 5 & - & ACR- 03 & & & & & & & & & & & & & & & & & & \\
\hline 1.5 & $\mathrm{~K} 4 \mathrm{M}$ & 10 & - & ACR- 04 & & & & & & & & & & & & & & & & & & \\
\hline 1.5 & $\mathrm{~K} 15 \mathrm{M}$ & 10 & - & ACR- 05 & & & & & & & & & & & & & & & & & & \\
\hline 1.5 & K100M & 10 & - & ACR- 06 & & & & & & & & & & & & & & & & & & \\
\hline 1.5 & $\mathrm{~K} 4 \mathrm{M}$ & 20 & - & ACR- 07 & & & & & & & & & & & & & & & & & & \\
\hline 1.5 & $\mathrm{~K} 15 \mathrm{M}$ & 20 & - & ACR- 08 & & & & & & & & & & & & & & & & & & \\
\hline 1.5 & K100M & 20 & - & ACR- 09 & & & & & & & & & & & & & & & & & & \\
\hline 1.5 & $\mathrm{~K} 15 \mathrm{M}$ & 30 & - & ACR- 10 & 89.01 & \pm & 1.04 & 96.39 & \pm & 0.20 & & & & & & & & & & & & \\
\hline 1.5 & K100M & 30 & - & ACR- 11 & 86.09 & \pm & 1.10 & 93.25 & \pm & 1.41 & & & & & & & & & & & & \\
\hline 1.5 & $\mathrm{~K} 15 \mathrm{M}$ & 40 & - & ACR- 12 & 86.29 & \pm & 0.76 & 91.26 & \pm & 0.75 & 94.54 & \pm & 1.72 & 97.08 & \pm & 0.68 & 99.27 & \pm & 1.51 & & & \\
\hline 1.5 & K100M & 40 & - & ACR- 13 & 83.28 & \pm & 0.91 & 90.22 & \pm & 0.25 & 93.29 & \pm & 0.52 & 96.45 & \pm & 1.51 & 98.52 & \pm & 1.63 & & & \\
\hline 1.5 & $\mathrm{~K} 15 \mathrm{M}$ & 50 & - & ACR- 14 & 59.33 & \pm & 0.77 & 65.93 & \pm & 1.94 & 71.19 & \pm & 0.69 & 78.33 & \pm & 1.07 & 81.95 & \pm & 0.71 & 86.09 & \pm & 0.69 \\
\hline 1.5 & K100M & 50 & - & ACR- 15 & 57.39 & \pm & 0.89 & 63.51 & \pm & 2.02 & 69.38 & \pm & 0.59 & 75.01 & \pm & 0.32 & 80.36 & \pm & 0.61 & 83.66 & \pm & 0.95 \\
\hline 1.5 & $\mathrm{~K} 15 \mathrm{M}$ & 40 & 5 & ACR- 16 & 73.53 & \pm & 0.62 & 77.36 & \pm & 1.05 & 81.33 & \pm & 1.33 & 84.36 & \pm & 0.35 & 89.50 & \pm & 0.74 & 96.07 & \pm & 1.37 \\
\hline 1.5 & $\mathrm{~K} 100 \mathrm{M}$ & 40 & 5 & ACR- 17 & 71.33 & \pm & 0.40 & 76.09 & \pm & 1.03 & 80.01 & \pm & 0.81 & 82.11 & \pm & 1.72 & 85.07 & \pm & 1.48 & 92.51 & \pm & 0.84 \\
\hline 1 & $\mathrm{~K} 15 \mathrm{M}$ & 40 & - & ACR- 18 & 83.98 & \pm & 1.02 & 88.64 & \pm & 1.41 & 90.32 & \pm & 0.71 & 92.99 & \pm & 0.93 & 95.82 & \pm & 1.46 & 97.87 & \pm & 1.08 \\
\hline 5 & $\mathrm{~K} 15 \mathrm{M}$ & 40 & - & ACR- 19 & 50.73 & \pm & 1.32 & 58.87 & \pm & 1.05 & 64.53 & \pm & 1.08 & 70.43 & \pm & 1.19 & 74.39 & \pm & 0.45 & 81.85 & \pm & 1.22 \\
\hline 10 & $\mathrm{~K} 15 \mathrm{M}$ & 40 & - & ACR- 20 & 27.47 & \pm & 0.66 & 30.73 & \pm & 1.22 & 35.57 & \pm & 1.50 & 40.36 & \pm & 0.40 & 48.50 & \pm & 0.52 & 52.42 & \pm & 1.25 \\
\hline 1.5 & - & - & - & RT & 70.49 & \pm & 0.78 & 75.98 & \pm & 1.24 & 82.95 & \pm & 1.97 & 85.47 & \pm & 0.71 & 89.21 & \pm & 1.32 & 97.07 & \pm & 0.77 \\
\hline
\end{tabular}




\section{Results and Discussion}

\section{Evaluation of Physiochemical properties of Controlled release Alprazolam Tablets}

Each formulated tablets were characterized by various physico-chemical properties such as weight variation, hardness, thickness, friability, assay and content uniformity. The average tablet weight was found to be in the $217-240 \mathrm{mg}(230 \mathrm{mg})$ target. The average tablet hardness was $5.0-9.5 \mathrm{~kg}\left(8 \mathrm{~kg} / \mathrm{cm}^{2}\right.$ target $)$. Content uniformity of tablet was $91.92-109.94$ $\%$ and assay of tablet was $96.32-103.30 \%$. The results are given in Table 2.

\section{In Vitro Drug Release}

Dissolution test of reference tablet and all tablets formulated by wet granulation were conducted at $37{ }^{\circ} \mathrm{C}$ using USP Apparatus 2 (Paddle) at a rotation speed of $50 \mathrm{RPM}$. The dissolution medium consisted of $500 \mathrm{ml}$ of $0.1 \mathrm{~N} \mathrm{HCl}$ solution $(\mathrm{pH}=1.1)$. A $10 \mathrm{ml}$ sample was taken from the medium at 1, 2, 3, 4, 5, 6, 7, 9, 10, 11 and $12 \mathrm{~h}$. and equal volume of medium was replaced into each of the dissolution jars after each sampling. Each sample was filtered through $10 \mu \mathrm{m}$ filter prior to analysis. The amount of drug release was determined by ultraviolet spectroscopy [10].The dissolution release specification were as follows: less than $45 \%$ release at $2 \mathrm{~h}, 45-65 \%$ release at $4 \mathrm{~h}, 75-90 \%$ release at $8 \mathrm{~h}$, and greater than $90 \%$ release at $12 \mathrm{~h}$ [38]. The dissolution profile of marketed tablets tested by using USP dissolution apparatus II (paddle) at $37 \pm 2^{\circ} \mathrm{C}$ and $50 \mathrm{rpm} 500 \mathrm{ml} 0.1 \mathrm{~N} \mathrm{HCl}$ was shown in Figure1.

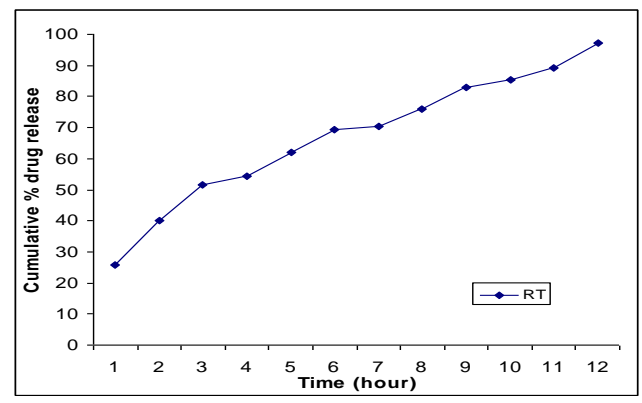

Figure1 Drug release profile of marketed SR Alprazolam Tablet.

\section{Effect of Polymer concentration}

\section{HPMC K4M}

In tablet formulations containing hydrophilic polymers like HPMC, the release of active drug is controlled by the rate of formation of a partially hydrated gel layer of the tablet surface formed upon contact with aqueous gastric media following ingestion and the continuous formation of additional gel layers [38].Formulation ACR-01, ACR-04, ACR-07 contained HPMC K4M in increasing order Figure 2

A comparison of their dissolution profile is given in

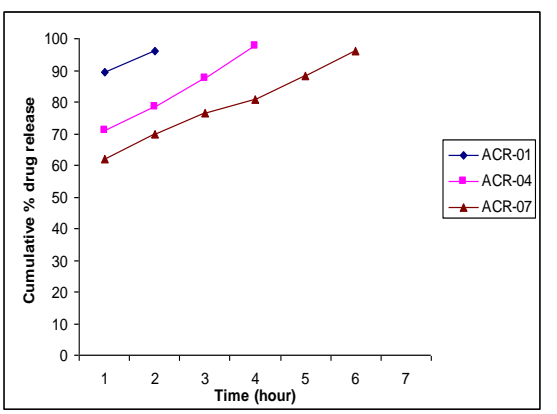

Figure 2 Drug release profile of formulation ACR 01, ACR 04 and ACR 07 using 5\%, $10 \%$ and $20 \%$ HPMC K4M as polymer respectively.

This profile indicates that increase in percentage of HPMC increases the dissolution time. The difference in drug release profile is clearly observed. In formulation ACR 01 
about $96 \%$ of drug was released within 2 hour which contains only $5 \%$ HPMC K4M of the total weight of Tablet. In formulation ACR 04 about $98 \%$ of drug was released within 4 hours and this formulation contains exactly double concentration of polymer than the formulation ACR 01. In Formulation ACR- 07 Containing $30 \%$ of HPMC K4M about $96 \%$ of drug was released within 6 hours.

\section{HPMC K15M}

Formulation ACR- 02, ACR-05, ACR-08, ACR-10, ACR-12, and ACR- 14 contained $5 \%$, $10 \%, 20 \%, 30 \%, 40 \%$ and $50 \%$ HPMC K15M respectively of the total weight of tablets. A comparison of their dissolution profile is given in Figure 3.

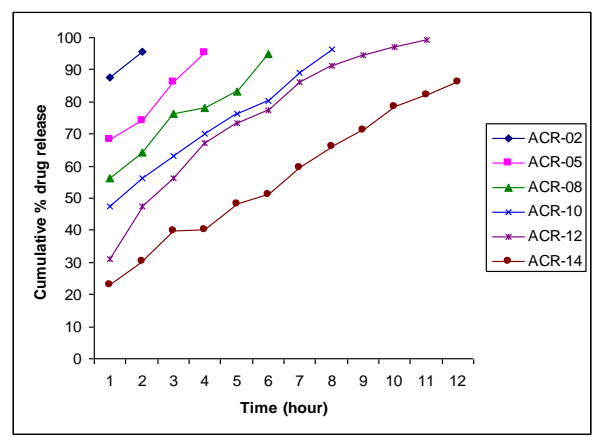

Figure 3 Drug release profile of formulation ACR 02, ACR 05, ACR 08, ACR 10 and ACR 12 using $5 \%, 10$ $\%, 20 \%, 30 \%, 40 \%$ and $50 \%$ HPMC K15M as polymer respectively.

In formulation ACR-02 about $95 \%$ of drug was released within 2 hour, in formulation ACR05 about $95 \%$ of drug was released within 4 hours, in Formulation ACR-08 about $96 \%$ of drug was released within 6 hours, in formulation ACR-10 about $96 \%$ of drug was released at 8 hour and in formulation ACR-12 about $99 \%$ of drug was released at 11 hour and in formulation ACR-14 about $86.0 \%$ of drug was released which indicates that increase in percentage of HPMC increases the dissolution time. The release of the drug was found to be appreciable in the case of formulation ACR-12.

\section{HPMC K100M}

Formulation ACR- 03, ACR-06, ACR-09, ACR-11, ACR-13 and ACR-14 contained $5 \%, 10$

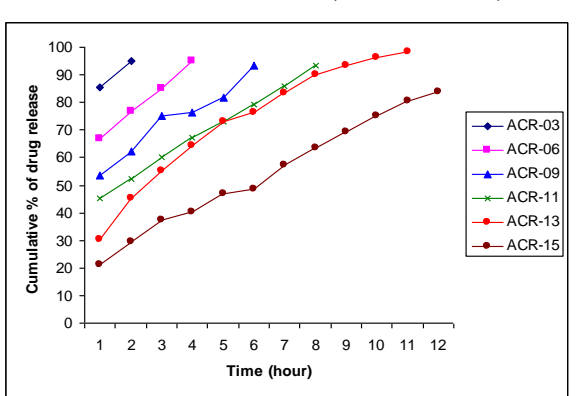
$\%, 20 \%, 30 \%, 40 \%$ and 50\% HPMC K100M respectively of the total weight of tablets. A comparison of their dissolution profile is given in Figure 4

Figure 4 Drug release profile of formulation ACR-03, ACR-06, ACR-09, ACR-11, ACR-13 and ACR-15 using $5 \%, 10 \%, 20 \%, 30 \%, 40 \%$ and $50 \%$ HPMC K100M as polymer respectively.

In formulation ACR-03 about $95 \%$ of drug was released within 2 hour, in formulation ACR06 about $95 \%$ of drug was released within 4 hours, in Formulation ACR-09 about $93 \%$ of drug was released within 6 hours, in formulation ACR-11 about $93 \%$ of drug was released at 8 hour and in formulation ACR-13 about $98 \%$ of drug was released at 11 hour and in formulation ACR-15 about $84 \%$ of drug released at 12 hour, the release of the drug was found to be appreciable in the case of formulation ACR-13. which indicates that increase in percentage of HPMC increases the dissolution time and retards the drug release. The difference in drug release profile is clearly observed. 


\section{Effect of various types Hydrophilic polymer at same concentration}

Drug release from the formulation ACR-01, ACR-04, ACR-07 using HPMC K4M, formulation ACR-02, ACR-05, ACR-08, ACR-10, ACR-12, ACR-14 using HPMC K15M and formulation ACR-03, ACR-06, ACR-09, ACR-11, ACR-13, ACR-15 using HPMC $\mathrm{K} 100 \mathrm{M}$ were found to be in different even Same concentration of HPMC were incorporated in these formulation. These formulation indicates that increasing polymer concentration retard the drug release. The drug release profiles of these formulations have been shown in Figure given below.

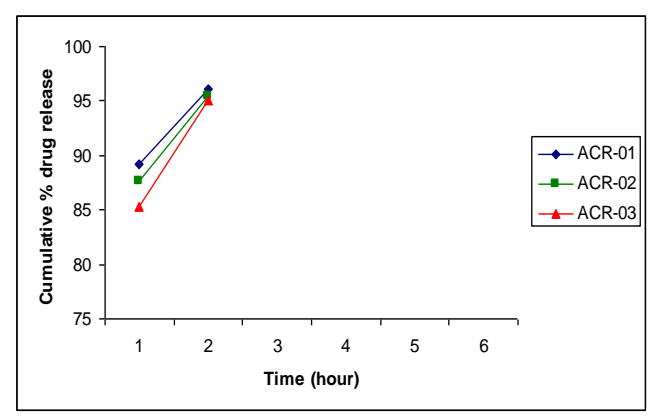

Figure 5 Drug release profile of formulation ACR 01

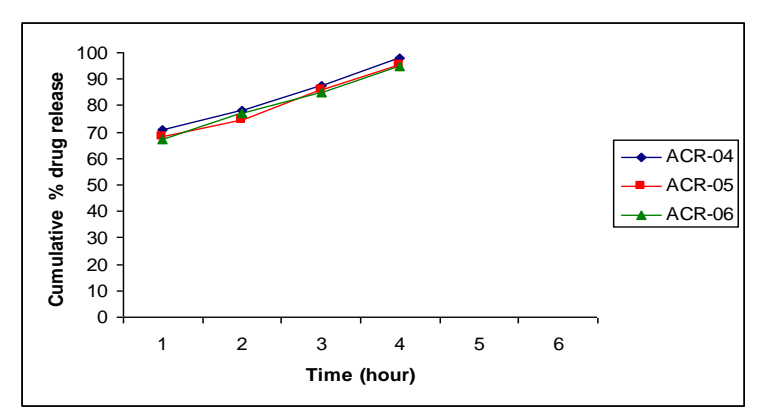

Figure 6 Drug release profile of formulation ACR 04, ACR 02 and ACR 03 using 5\% HPMC K4M, HPMC K15M ACR 05 \& ACR 06 using $10 \%$ HPMC K4M, HPMC K15 M and HPMC K100M respectively.
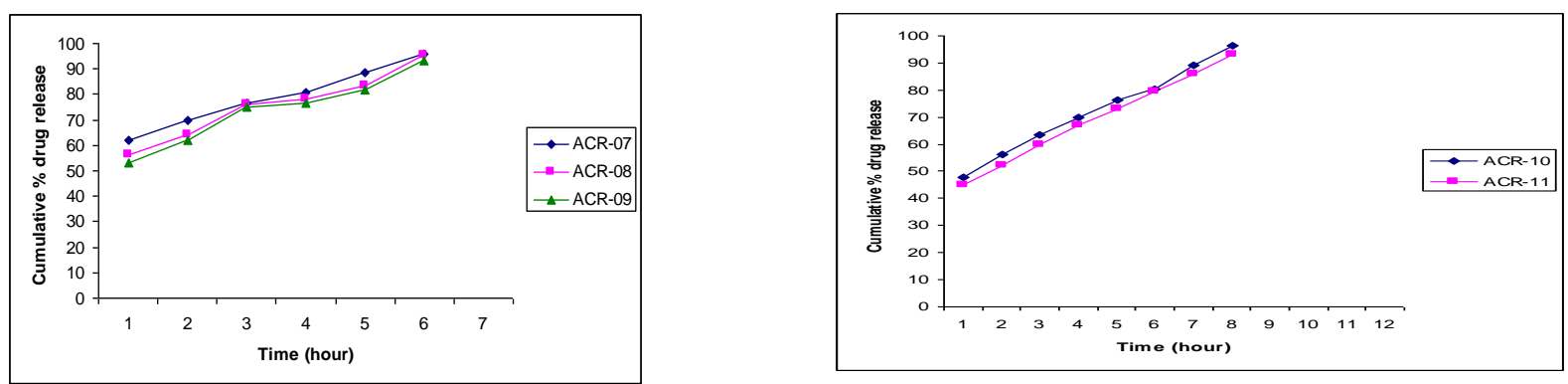

Figure 7 Drug release profile of formulation ACR 07 Figure 8 Drug release profile of formulation ACR 10, ACR ACR 08 \& ACR 09 using 20\% HPMC K4M, HPMC K15M 11 using $30 \%$ HPMC K15 M and HPMC K100M respectively. \& HPMC K100M respectively.
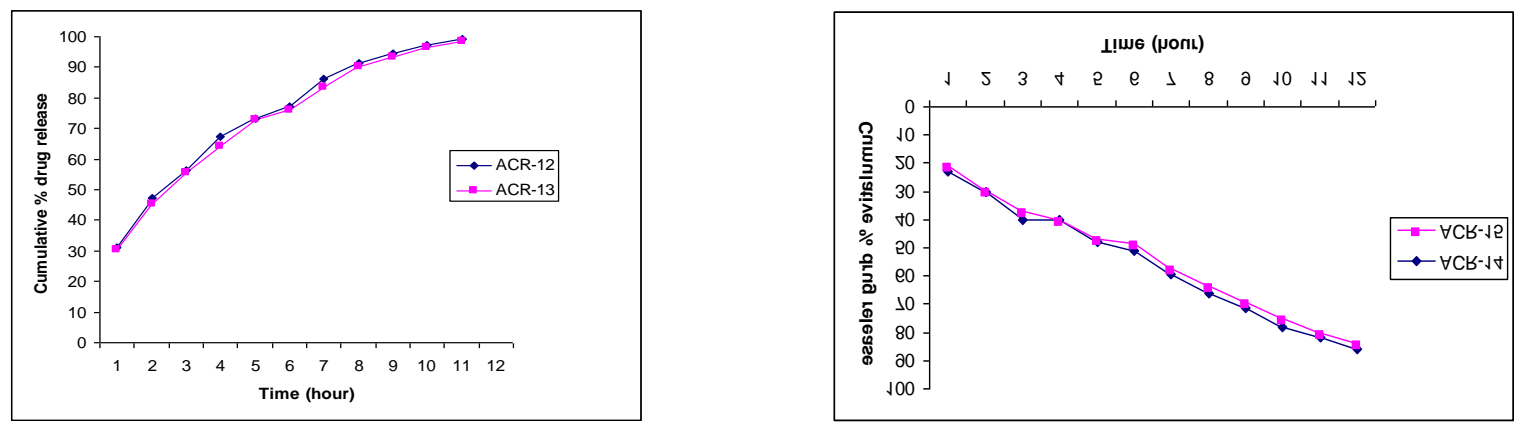

Figure 9 Drug release profile of formulation ACR 12 Figure 10 Drug release profile of formulation ACR 14, ACR 15 and ACR 13 using $40 \%$ HPMC K15M, HPMC K100M using $50 \%$ HPMC K4M, HPMC K15 M and HPMC K100M respectively. respectively.

\section{Hydrophilic with Hydrophobic polymer}

The dissolution profiles of various formulation (ACR-16 and ACR-17) by using $40 \%$ hydrophilic polymer (HPMC K15M, HPMC K100M), in combination with hydrophobic 
polymer, $5 \%$ EC respectively are shown in Figure 3.12. In these formulations low concentration of Ethyl cellulose was incorporated in the formulation containing HPMC to see the change in the release profile of the drug from the matrix. Incorporation of Ethyl cellulose was found to control the drug release. In formulation ACR-16 about $96 \%$ of drug was released within 12 hour and in formulation ACR-17about $92 \%$ of drug was released within 12 hours,

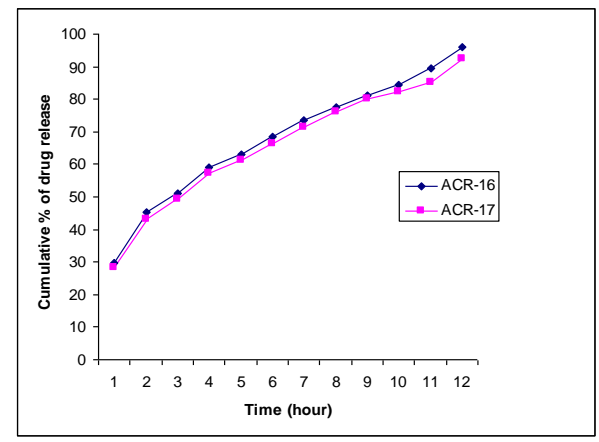

Figure 11 Drug release profile of formulation ACR-16 and ACR-17 using HPMC K15 M (40\%) and HPMC K100M (40\%) in Combination with $5 \%$ EC respectively.

\section{Effect of Drug loading on release profile}

Formulation ACR-18, ACR-12, ACR-19, ACR-20 and ACR-21 contained 1.0, 1.5, 5.0, 10.0 and $15.0 \mathrm{mg}$ Alprazolam respectively and each formulation contained $40 \% \mathrm{HPMC}$ K15M.Dissolution study was performed to see the effect of drug loading on release profile of these formulations and have been shown in following Figure which indicates that increasing the loading dose of alprazolam causes decrease in drug release having constant HPMC concentration.

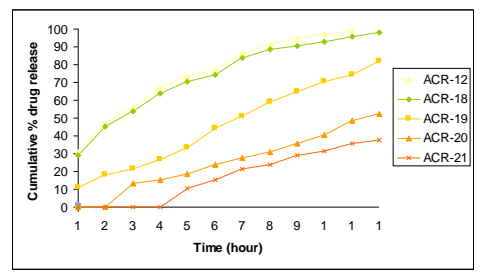

Figure 12 Drug release profile of formulation ACR 12, ACR 18, ACR 19, ACR 20 and ACR 21 containing $1.0 \mathrm{mg}, 1.5 \mathrm{mg}$, $5 \mathrm{mg}, 10 \mathrm{mg}$, and $15 \mathrm{mg}$ alprazolam.

\section{Comparison of dissolution profile of formulated product with marketed product}

In formulation ACR-16 drug released was about $29 \%$ in 1 hour and $96 \%$ within 12 hour and in formulation ACR-17 drug released was about $28 \%$ in 1 hour and about $92 \%$ within 12 hours. Similarly, in marketed Tablet drug released was $26 \%$ in 1 hour and $97 \%$ within 12 hours. And drug release profile of these formulated and marketed products can be compared from the following Figure.

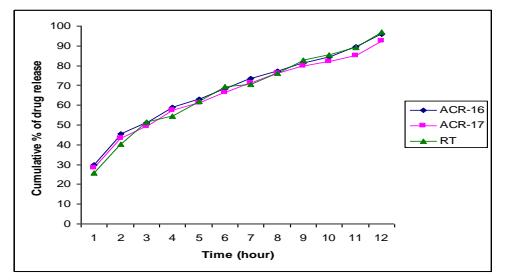

Figure 13 comparison of Drug release profile of formulated product ACR-16 and ACR-17 with marketed Product.

\section{Drug Release Kinetics}

The drug release data from the formulated and marketed tablets were fitted to various kinetics equations following Zero order, First order, Higuchi model and Korsmeyer-Peppas 
equations.and presented in Table 4. The correlation coefficient obtained from curve fitting into different kinetic equation suggested that most of the formulations were found to follow either Higuchi or Korsmeyer-peppas model and some of formulation were found to follow Zero order and 1 st order. The release exponent " $n$ " value for the different formulations ranged from $0.48-1.41$. The release kinetics from reference Tablets was best fitted with Higuchi model with $\mathrm{R}^{2}$ value 0.9916 . Formulation ACR-04 and ACR-20 were found to follow $1^{\text {st }}$ order kinetics with $\mathrm{R}^{2}$ value 0.9993 and 0.989 respectively. Only formulation ACR-19 was found to follow Zero order kinetics with $\mathrm{R}^{2}$ value 0.9895.Regression coefficient of drug release profiles of ACR-12, ACR-13, ACR-14, ACR-15, ACR-16 and ACR-17 obtained with Higuchi model are all greater than 0.96 .

The release exponents " $n$ " of these six formulations are in between 0.6836 to 0.8614 followed anomalous transport $(0.5<\mathrm{n}<1.0)$ indicating both diffusion and swelling controlled release [11]. Regression coefficient of drug release profiles of ACR-05 ACR-06, ACR-07, ACR-08, ACR-09, ACR-10, ACR-11, ACR-18 and ACR-21 obtained with Korsmeyer Peppas model are all above 0.96 . The release exponent " $n$ " values of these formulations are in between 0.48 to 0.64 except formulation ACR- 21 with $n$ value 1.41 showed Super-Case-II transport [12]. The formulations ACR-18 showed $\mathrm{n}$ value less than 0.5 . The drug diffusion partially through a swollen matrix and water-filled pores in the formulations could be the reason for smaller value of release exponent $(\mathrm{n}<0.5)$.

\section{Statistical Analysis}

One way ANOVA was applied to the various formulated tablets to find out any significant difference between release profiles of these formulations. The formulation ACR-12 and ACR-13 containing $40 \%$ HPMC K15M and $40 \%$ HPMC K100M with dissolution profile up to 11 hours was tested and found that there is no significant difference ( $p>0.05)$ in drug release rate between these two formulations. Similarly formulation ACR-16, ACR-17 containing $40 \%$ HPMC K15M and $40 \%$ HPMC K100M with $5 \%$ EC on both formulation with dissolution profile up to 12 hours was tested and found that no significant difference ( $\mathrm{p}>$ 0.05 ) in drug release rate was found between formulation ACR-16 and ACR- 17.

\section{Similarity and Dissimilarity Factor}

Models independent methods were used to test the dissimilarity factor $\mathrm{f}_{1}$ and Similarity factor $\mathrm{f}_{2}$ of the various formulated and reference tablets. According to this method, a product is considered to be similar when its $\mathrm{f}_{2}$ value lies within 50-100 and $\mathrm{f}_{1}$ within $0-15$. When formulation ACR-12 and ACR-13 were compared by similarity factor and value was found to be 79.07 and it indicates that there is no significant difference between formulation ACR-12 and ACR-13.Similarly, Similarity factor between formulation ACR-16 and ACR-17 was 70.935 and it also indicates that there is no significant difference between these formulations.

Finally formulation ACR-16 was compared with reference tablet with similarity factor and value was assessed by 68.93 which indicate that there is no significant difference between these two formulations. Dissimilarity factor $\mathrm{f}_{1}$ between formulation ACR-12 and ACR-13 was calculated and value was found to be 1.837 which indicates there is no significant difference between these two formulations. Formulation ACR-16 and ACR-17 was also tested by dissimilarity factor and value was found to be 3.227 and found that there no significant difference between these two formulation. Finally formulation ACR-16 and reference tablet was compared by dissimilarity factor and value was 3.053 and it also indicates that there is no significant difference between these two formulations. 
Table 4 Drug release kinetics of formulated and marketed controlled release Alprazolam tablets

\begin{tabular}{l|llllllll}
\hline & \multicolumn{2}{|l}{ Zero Order Model } & \multicolumn{2}{l}{ First Order Model } & \multicolumn{2}{l}{ Higuchi Model } & \multicolumn{2}{l}{ Peppas Model } \\
Formulation & $\mathrm{k}$ & $\mathrm{R}^{2}$ & $\mathrm{k}$ & $\mathrm{R}^{2}$ & $\mathrm{k}$ & $\mathrm{R}^{2}$ & $\mathrm{n}$ & $\mathrm{R}^{2}$ \\
& $\left(\% \mathrm{hr}^{-1}\right)$ & & $\left(\% \mathrm{hr}^{-1}\right)$ & & $\left(\% \mathrm{hr}^{-1}\right)$ & & & \\
\hline ACR 04 & 29.369 & -5.0831 & 0.1080107 & 0.9993 & 52.867 & 0.9711 & 0.5037 & 0.9978 \\
ACR 05 & 28.503 & -4.1164 & 0.1146894 & 0.9903 & 51.232 & 0.9606 & 0.5181 & 0.9981 \\
ACR 06 & 28.496 & -4.3691 & 0.1139985 & 0.9951 & 51.252 & 0.9897 & 0.543 & 0.9971 \\
ACR 07 & 19.483 & -3.7954 & 0.0840595 & 0.991 & 42.287 & 0.9805 & 0.5636 & 0.9954 \\
ACR 08 & 18.817 & -2.0623 & 0.0983381 & 0.9536 & 40.676 & 0.9624 & 0.575 & 0.9929 \\
ACR 09 & 18.414 & -1.673 & 0.103635 & 0.9433 & 39.754 & 0.963 & 0.5965 & 0.9923 \\
ACR 10 & 14.156 & -0.5509 & 0.0958048 & 0.9793 & 34.786 & 0.9843 & 0.6111 & 0.9888 \\
ACR 11 & 13.668 & -0.2838 & 0.1015623 & 0.9823 & 33.51 & 0.9869 & 0.6478 & 0.9889 \\
ACR 12 & 11.156 & 0.3313 & 0.1001805 & 0.8451 & 31.676 & 0.988 & 0.6836 & 0.9716 \\
ACR 13 & 10.984 & 0.4003 & 0.1029441 & 0.8537 & 31.141 & 0.9916 & 0.7113 & 0.9709 \\
ACR 14 & 5.7502 & 0.7948 & 0.1123864 & 0.9512 & 23.365 & 0.9753 & 0.8258 & 0.9663 \\
ACR 15 & 8.0178 & 0.8126 & 0.1149197 & 0.945 & 22.63 & 0.9787 & 0.8614 & 0.9617 \\
ACR 16 & 9.3592 & 0.2456 & 0.0868231 & 0.8739 & 27.735 & 0.9933 & 0.727 & 0.9682 \\
ACR 17 & 9.0622 & 0.2586 & 0.0877443 & 0.867 & 26.855 & 0.9935 & 0.7513 & 0.969 \\
ACR 18 & 10.157 & 0.2981 & 0.0925806 & 0.8242 & 30.102 & 0.9762 & 0.4828 & 0.9874 \\
ACR 19 & 7.0229 & 0.9895 & 0.172725 & 0.9356 & 19.983 & 0.8457 & 0.8449 & 0.9839 \\
ACR 20 & 4.0958 & 0.9625 & 0.1549919 & 0.989 & 11.414 & 0.7313 & 1.0261 & 0.9814 \\
ACR 21 & 2.9274 & 0.8708 & 0.1711129 & 0.9207 & 7.9462 & 0.6002 & 1.4164 & 0.9719 \\
RT & 9.3125 & 0.4936 & 0.1239014 & 0.8616 & 27.461 & 0.9916 & 0.5004 & 0.9905 \\
\hline
\end{tabular}

\section{Conclusion}

- The release of Controlled release alprazolam tablet depends on the various viscosity grade of HPMC. Increasing the viscosity of polymer retards the drug release.

- Drug release from HPMC-K4M > HPMC-15M > HPMC-K100M.

- Increase in concentration of polymer increases the dissolution time.

- When Aplrazolam loading was increased maintaining HPMC concentration constant the drug release was found to be decreased.

- In formulation when EC in combination with water-soluble HPMC was used the release was found to be decreased. 
Acknowledgement

Authors are grateful to the Lomus Pharmaceuticals Pvt. Ltd for providing the total facilities for this research work by supporting with Raw materials as well as by equipments and facility.

\section{REFERENCES}

1 Hui HW, Lee VHL, Robinson JR. Design and fabrication of oral controlled release drug delivery systems. Controlled Drug Delivery Fundamentals and Applications $2^{\text {nd }}$ ed. 1987: p. 373-432. New York].

2 Ali J, Khar RK, Ahuja A. A test book of pharmaceutics and pharmacokinetics, 2002: p. $204-272$.

3 Chiao CSL, Robinson JR. Sustained - Release Drug Delivery Systems.In: Gennaro AR. $19^{\text {th }}$ ed. Remington; The Science and Practice of Pharmacy 1995: p. $1660-$ 1675. Pennsylvania: Easton.

4 Mario Grassi and Gabriele Grassi. Mathematical Modeling and Controlled Drug Delivery: Matrix Systems Current Drug Delivery, 2005,2,97 - 116 Germany.

5 Rodriguez C. F., Bruneau N., Barra J., Alfonso D., and Doelker E. Hydrophilic Cellulose Derivatives as Drug Delivery Carriers: Influence of Substitution Type on the properties of Compressed Matrix Tablets. In: Wise D.L. et al ed. Handbook of Pharmaceutical controlled Release Technology 2000.pl. New York, NY: Marcel Dekker Inc.

$6 \quad$ www.inchem.org

7 Donald L. Wise, Handbook of pharmaceutical controlled release technology, Marcel Dekker , INC, 2002

8 Crowley M.M., Schroeder B., Federsdorf A., Obara S., Talarico M., Kucera S., McGinity J.W. Physicochemical Properties and Mechanism of Drug Release From Ethylcellulose Matrix Tablets Prepared By Direct Compression and Hot-melt Extrusion .International Journal of Pharmaceutics 2004, 269: 509 - 522.

9 .file:///E|/alprazolam/Alprazolam - Article.htm

10 Vorapann Mahaguna, Robert L. Talbert, Jay I. Peters, Sandra Adams, Thomas D. Reynolds, Francis Y. W. Lam, Robert O. Williams III. Influence of HPMC polymer on in vitro and in vivo performance of controlled release tablets containing Alprazolam, European Journal of Pharmaceutics and Biopharmaceutics 56 (2003) 461-468.

11 J. Siepmann, N. A. Peppas, 2001. Modeling of drug release from delivery systems based on hydroxy propyl methyl cellulose (HPMC), Adv. Drug Del. Reviews. 48:139157(2001).

12 N. A. Peppas, 1985. Analysis of fickian and non-fickian drug release from polymers. Pharm. Actahelv. 60(4): 110-111. 